International Journal of Pure and Applied Mathematics

Volume 101 No. 1 2015, 101-108

ISSN: 1311-8080 (printed version); ISSN: 1314-3395 (on-line version)

url: http://www.ijpam.eu

doi: http://dx.doi.org/10.12732/ijpam.v101i1.11

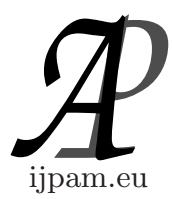

\title{
THE HEAVIEST WEIERSTRASS $N$-SEMIGROUPS ON NON-HYPERELLIPTIC CURVES?
}

\author{
E. Ballico \\ Department of Mathematics \\ University of Trento \\ 38123 Povo (Trento) - Via Sommarive, 14, ITALY
}

\begin{abstract}
We propose the two "heaviest" Weierstrass n-semigroups for nonhyperelliptic curves (one on trigonal curves with maximal Maroni invariant and with totally ramification points and one on bielliptic curves and with as points some of the ramification points). We compute them when $n=2$.
\end{abstract}

AMS Subject Classification: $14 \mathrm{H} 55$

Key Words: Weierstrass $n$-semigroup, semigroup of non-gaps, bielliptic curve, trigonal curve

\section{The Heaviest $n$-semigroups?}

Let $X$ be a smooth and connected projective curve of genus $g \geq 4$ defined over an algebraically closed field $\mathbb{K}$ with characteristic zero. Fix $P_{1}, \ldots, P_{n} \in X$ such that $P_{i} \neq P_{j}$ for all $i \neq j$. Let $H\left(P_{1}, \ldots, P_{n}\right) \subset \mathbb{N}^{n}$ be the set of all $n$-ples $\left(a_{1}, \ldots, a_{n}\right) \in \mathbb{N}^{n}$ such that there is a rational function on $X$ with $a_{1} P_{1}+\cdots+$ $a_{n} P_{n}$ as its divisor of poles ([1], [2], [3]) The set $H\left(P_{1}, \ldots, P_{n}\right)$ is a semigroup (called the Weierstrass semigroup of $P_{1}, \ldots, P_{n}$ ) for the componentwise addition $+: \mathbb{N}^{n} \times \mathbb{N}^{n} \rightarrow \mathbb{N}^{n}$. The elements of the finite set $G\left(P_{1}, \ldots, P_{n}\right):=\mathbb{N}^{n} \backslash$ $H\left(P_{1}, \ldots, P_{n}\right)$ are called the gaps of $\left(P_{1}, \ldots, P_{n}\right)$. Note that the genus $g$ is

Received: March 6, 2015

(c) 2015 Academic Publications, Ltd. url: www.acadpubl.eu 
obtained from $H\left(P_{1}, \ldots, P_{n}\right)$ by the formula $g=\sharp\left((\mathbb{N} \times(0, \ldots, 0)) \cap\left(\mathbb{N}^{n} \backslash\right.\right.$ $\left.H\left(P_{1}, \ldots, P_{n}\right)\right)$. There is a definition of weight $w\left(P_{1}, \ldots, P_{n}\right)$ of the semigroup $H\left(P_{1}, \ldots, P_{n}\right)([1$, Definition 0.1$])$; set

$$
w\left(P_{1}, \ldots, P_{n}\right):=\sum_{\left(a_{1}, \ldots, a_{n}\right) \in \mathbb{N}^{n}} h^{1}\left(\mathcal{O}_{X}\left(a_{1} P_{1}+\cdots+a_{n} P_{n}\right)\right)-\left(\begin{array}{l}
g+n \\
n+1
\end{array}\right) .
$$

We introduce here another definition of weight (we call it gist). Set

$$
v\left(P_{1}, \ldots, P_{n}\right):=\sum_{\left(a_{1}, \ldots, a_{\mathrm{n}}\right) \in H\left(P_{1}, \ldots, P_{\mathrm{n}}\right) \backslash\{0\}} h^{1}\left(\mathcal{O}_{X}\left(a_{1} P_{1}+\cdots+a_{n} P_{n}\right)\right) .
$$

We would like to find the n-semigroups with maximal weight and maximal gist (for fixed $g, n$ ) among the non-hyperelliptic curves (for the elliptic curves see [1]). It is better to separate the problem if we allow also curves which are covering of a curve of positive genus. Among the curves covering a positive genus curve the maximal weight and gist should be on bielliptic curves, but with certain restrictions. We discuss these restrictions in Example 3, computing $H\left(P_{1}, P_{2}\right)$ and $v\left(P_{1}, P_{2}\right)$. In the second case it should be with $X$ trigonal (see Examples 1 and 2 in which we compute $H\left(P_{1}, P_{2}\right)$ and $v\left(P_{1}, P_{2}\right)$ ). These examples make sense only if $g$ is not too small with respect to $n$. Are these cases maximal for the weight or for the gist? It is easier to compute the gist than the weight, but they are known if we know $H\left(P_{1}, \ldots, P_{n}\right)$, because the structure of $H\left(P_{1}, \ldots, P_{n}\right)$ with the partial order $\left(a_{1}, \ldots, a_{n}\right) \leq\left(b_{1}, \ldots, b_{n}\right) \Leftrightarrow$ $a_{i} \leq b_{i}$ for all $i$ uniquely determines all integers $h^{1}\left(\mathcal{O}_{X}\left(a_{1} P_{1}+\cdots+a_{n} P_{n}\right)\right)$, $a_{i} \geq 0$

We may also ask for $X, P_{1}, \ldots, P_{n}$ with very high $w\left(P_{1}, \ldots, P_{n}\right)$ and $v\left(P_{1}\right.$, $\left.\ldots, P_{n}\right)$ with the additional condition that no $P_{i}$ is a Weierstrass point of $X$, i.e. $G\left(P_{i}\right)=1, \ldots, g-1$ for all $i$. If $n$ is even, it is easy to see that the extremal $X, P_{1}, \ldots, P_{n}$ are obtained on hyperelliptic curves with $P_{1}, \ldots, P_{n}$ invariant for the hyperelliptic involution. I If $n$ is odd the obvious candidate is computed (see Example 4).

Example 1. Fix an even integer $g \geq 6$. Let $X$ be a genus $g$ trigonal curve which is cyclic $([4, \S 3])$ and with Maroni invariant $g / 2-1$ ([7, page 172$]$. Call $R \in \operatorname{Pic}^{3}(X)$ the trigonal line bundle. Since $X$ has Maroni invariant $g / 2-1$, $h^{0}\left(R^{\otimes t}\right)=t+1$ if $0 \leq t \leq g / 2$ and $h^{1}\left(R^{\otimes t}\right)=0$ if $t \geq g / 2([7$, page 172]. We call $P_{1}, \ldots, P_{g+2}$ the ramification points of the trigonal covering $X \rightarrow \mathbb{P}^{1}$. $X$ is the smooth model of the curve $y^{3}=\prod_{i=1}^{g+2}\left(x-t_{i}\right)$ with $P_{i}=\left(t_{i}, 0\right) \in \mathbb{K}^{2}$ and $t_{i} \neq t_{j}$ for all $i \neq j([4$, Remark 3.4']). By [4, Case 3) at page 246]), $G\left(P_{i}\right)=(1,2,4,5, \ldots, 3 g / 2-1,3 g / 2+1)$. We only take n-semigroups with 
$n \leq g+2$ and take as points some of the points $P_{1}, \ldots, P_{g+2}$. With these restrictions for any $a=\left(a_{1}, \ldots, a_{n}\right) \in \mathbb{N}^{n}$ with $a_{i} \equiv 0(\bmod 3)$ for all $i$ we know that $a \in H\left(P_{1}, \ldots, P_{n}\right)$ and the integers $h^{i}\left(\mathcal{O}_{X}\left(a_{1} P_{1}+\cdots+a_{n} P_{n}\right)\right), i=0,1$, but we also know it in most other cases. Write $a_{i}=3 b_{i}+e_{i}$ with $0 \leq e_{i} \leq 2$. Since $h^{1}\left(R^{\otimes g / 2}\right)=0$, if $c:=b_{1}+\cdots+b_{n} \geq g / 2$, then $a \in H\left(P_{1}, \ldots, P_{n}\right)$ and $h^{1}\left(\mathcal{O}_{X}\left(a_{1} P_{1}+\cdots+a_{n} P_{n}\right)\right)=0$. Now assume $c<g / 2$. We have the complete picture only for 2-semigroups and hence we take $n=2$ and $e_{1}+e_{2}>0$. It is sufficient to classify all cases with $e_{1} \geq e_{2}$.

(a) Assume $1 \leq e_{1} \leq 2$ and $e_{2}=0$. We have $\mathcal{O}_{X}\left(a_{1} P_{1}+a_{2} P_{2}\right) \cong R^{\otimes c}\left(e_{1} P_{1}\right)$. The gap sequence of $P_{1}$ gives $\left(a_{1}, a_{2}\right) \in H\left(P_{1}, P_{2}\right)$ if and only if $c=g / 2-1$ and $e_{1}=2$ and in this case $h^{1}\left(\mathcal{O}_{X}\left(a_{1} P_{1}+a_{2} P_{2}\right)\right)=0$. Indeed by $[4$, line 15 of page 253] the condition that $R$ has Maroni invariant $g / 2-1$ implies that each $P_{i}$ has type I in the sense of [4, Definition 2.13] and hence that all integers $x \geq 3(g / 2-1)+2$ are contained in $H\left(P_{i}\right)$.

(b) Assume $e_{1}=e_{2}=1$. Since $c<g / 2$, part (a) gives $\left(a_{1}-1, a_{2}\right) \notin$ $H\left(P_{1}, P_{2}\right)$ and $\left(a_{1}, a_{2}-1\right) \notin H\left(P_{1}, P_{2}\right)$. Therefore $\left(a_{1}, a_{2}\right) \in H\left(P_{1}, P_{2}\right)$ if and only if $h^{0}\left(\mathcal{O}_{X}\left(a_{1} P_{1}+a_{2} P_{2}\right)\right)=c+2$.

Assume for the moment $c \leq g / 2-2$. We have $h^{0}\left(\mathcal{O}_{X}\left(\left(a_{1}+2\right) P_{1}+a_{2} P_{2}\right)\right)=$ $c+2$ and $P_{2}$ is the base locus of $\left|\mathcal{O}_{X}\left(\left(a_{1}+2\right) P_{1}+a_{2} P_{2}\right)\right|$, because $3 c+4 \in G\left(P_{2}\right)$. Therefore $h^{0}\left(\mathcal{O}_{X}\left(a_{1} P_{1}+a_{2} P_{2}\right)\right)=c+1$, i.e. $\left(a_{1}, a_{2}\right) \notin H\left(P_{1}, P_{2}\right)$.

Now assume $c=g / 2-1$. We have $\left(a_{1}+2, a_{2}+1\right) \in H\left(P_{1}, P_{2}\right)$ and $h^{0}\left(\mathcal{O}_{X}\left(\left(a_{1}+2\right) P_{1}+\left(a_{2}+1\right) P_{2}\right)\right)=c+3$, because $P_{2}$ has type I $([4$, Case 3$)$ at page 246]). Since $\left(a_{1}+2, a_{2}+1\right) \in H\left(P_{1}, P_{2}\right)$, we have $h^{0}\left(\mathcal{O}_{X}\left(\left(a_{1}+2\right) P_{1}+\right.\right.$ $\left.\left.a_{2} P_{2}\right)\right)=c+2$. We also know that $P_{2}$ is in the base locus of $\mathcal{O}_{X}\left(\left(a_{1}+2\right) P_{1}+\right.$ $\left.a_{2} P_{2}\right)$ ), because $P_{2}$ is of type I. Hence we cannot have $\left(a_{1}, a_{2}\right) \in H\left(P_{1}, P_{2}\right)$ and $h^{0}\left(\mathcal{O}_{X}\left(a_{1} P_{1}+a_{2} P_{2}\right)\right)=c+2$.

(c) Assume $e_{1}=2$ and $e_{2}=1$. First assume $c \leq g / 2-2$. We have $h^{0}\left(\mathcal{O}_{X}\left(\left(a_{1}+1\right) P_{1}+a_{2} P_{2}\right)\right)=c+2$ and $P_{2}$ is the base locus of $\mid \mathcal{O}_{X}\left(\left(a_{1}+1\right) P_{1}+\right.$ $\left.a_{2} P_{2}\right) \mid$. Therefore $h^{0}\left(\mathcal{O}_{X}\left(a_{1} P_{1}+a_{2} P_{2}\right)\right)=c+1$, i.e. $\left(a_{1}, a_{2}\right) \notin H\left(P_{1}, P_{2}\right)$.

Now assume $c=g / 2-1$. We have $\left.h^{0}\left(\mathcal{O}_{X}\left(a_{1}+1\right) P_{1}+a_{2} P_{2}\right)\right)=c+2$ and $P_{2}$ is a base point of $\left.\mathcal{O}_{X}\left(a_{1}+1\right) P_{1}+a_{2} P_{2}\right) \cong R^{\otimes g / 2}\left(P_{2}\right)$, because $P_{2}$ has type I. Hence $a \notin H\left(P_{1}, P_{2}\right)$.

(d) Assume $e_{1}=e_{2}=2$. First assume $c \leq g / 2-2$. Since $3 c+4$ and $3 c+5$ are gap-values of $P_{2}$, we have $h^{0}\left(\mathcal{O}_{X}\left(\left(a_{1}+1\right) P_{1}+a_{2} P_{2}\right)\right)=c+2$ and $2 P_{2}$ is the base divisor of $\left|\mathcal{O}_{X}\left(\left(a_{1}+1\right) P_{1}+a_{2} P_{2}\right)\right|$. Therefore $h^{0}\left(\mathcal{O}_{X}\left(a_{1} P_{1}+a_{2} P_{2}\right)\right)=c+1$, i.e. $\left(a_{1}, a_{2}\right) \notin H\left(P_{1}, P_{2}\right)$.

Now assume $c=g / 2-1$. Since $P_{2}$ has type I, we have $h^{1}\left(\mathcal{O}_{X}\left(\left(a_{1}+1\right) P_{1}+\right.\right.$ $\left.\left.a_{2} P_{2}\right)\right)=0, h^{0}\left(\mathcal{O}_{X}\left(\left(a_{1}+1\right) P_{1}+a_{2} P_{2}\right)\right)=g / 2+3$ and $\left(a_{1}+1, a_{2}\right) \in H\left(P_{1}, P_{2}\right)$. 
Hence $h^{0}\left(\mathcal{O}_{X}\left(a_{1} P_{1}+a_{2} P_{2}\right)\right)=g / 2+2$. Riemann-Roch gives $h^{1}\left(\mathcal{O}_{X}\left(a_{1} P_{1}+\right.\right.$ $\left.\left.a_{2} P_{2}\right)\right)=0$. Since $\left(a_{1}, a_{2}-1\right) \notin H\left(P_{1}, P_{2}\right)\left(\right.$ step $(\mathrm{c}),\left(a_{1}-1, a_{2}\right) \notin H\left(P_{1}, P_{2}\right)$ (step (c) with $\left(P_{2}, P_{1}\right)$ instead of $\left.\left(P_{1}, P_{2}\right)\right)$ and $h^{0}\left(\mathcal{O}_{X}\left(\left(a_{1}-1\right) P_{1}+\left(a_{2}-1\right) P_{1}\right)\right)=$ $c+1$ (step (a)), we get $\left(a_{1}, a_{2}\right) \in H\left(P_{1}, P_{2}\right)$.

Example 2. Fix an odd integer $g \geq 7$. Let $X$ be a genus $g$ trigonal curve which is cyclic $([4, \S 3])$ and with $n=(g-1) / 2$ in the sense of [4], i.e. with Maroni invariant $(g-1) / 2-1$. Call $R \in \operatorname{Pic}^{3}(X)$ the trigonal line bundle and let $f: C \rightarrow \mathbb{P}^{1}$ denote the trigonal covering. We have $f_{*}\left(\mathcal{O}_{C}\right) \cong$ $\mathcal{O}_{\mathbb{P}^{1}} \oplus \mathcal{O}_{\mathbb{P}^{1}}(-(g+1) / 2) \oplus \mathcal{O}_{\mathbb{P}^{1}}(-(g+3) / 2)$. Hence $h^{0}\left(R^{\otimes t}\right)=t+1$ if and only if $t \leq(g-1) / 2, h^{1}\left(R^{\otimes t}\right)=0$ if and only if $t \geq(g+1) / 2$ and in particular $h^{0}\left(R^{\otimes(g+1) / 2}\right)=(g+1) / 2+2$. We call $P_{1}, \ldots, P_{g+2}$ the ramification points of the trigonal covering $X \rightarrow \mathbb{P}^{1}$. By [4, Proposition 3.3] $X$ has $(g-1) / 2$ total ramification points of type II and we assume that $P_{1}, P_{2}$ are two of them to compute $H\left(P_{1}, P_{2}\right)$ and $v\left(P_{1}, P_{2}\right)$. Take $\left(a_{1}, a_{2}\right) \in \mathbb{N}^{2}$ and write $a_{i}=3 b_{i}+e_{i}$ with $b_{i} \in \mathbb{N}$ and $0 \leq e_{i} \leq 2$. Set $c:=b_{1}+b_{2}$. If $e_{1}=e_{2}=0$, then $\left(a_{1}, a_{2}\right)=$ $H\left(P_{1}, P_{2}\right)$ and we computed $h^{1}\left(\mathcal{O}_{X}\left(a_{1} P_{1}+a_{2} P_{2}\right)\right)=h^{1}\left(R^{\otimes c}\right)$. Hence we may assume $2 \geq e_{1} \geq e_{2}$ with $e_{1} \neq 0$. If $e_{2}=0$, then $a \in H\left(P_{1}, P_{2}\right)$ if and only if $3 c+e_{1} \in H\left(P_{1}\right)$. We know that this is the case if and only if either $c \geq(g+1) / 2$ with $h^{1}\left(\mathcal{O}_{X}\left(a_{1} P_{1}+a_{2} P_{2}\right)\right)=0$ or $c=(g-1) / 2$ and $e_{1}=1$ (with $h^{1}\left(\mathcal{O}_{X}\left(a_{1} P_{1}+a_{2} P_{2}\right)\right)=0$ ), because $P_{1}$ has type I. If $e_{1} \geq e_{2}>0$, we have $a \in H\left(P_{1}, P_{2}\right)$ and $h^{1}\left(\mathcal{O}_{X}\left(a_{1} P_{1}+a_{2} P_{2}\right)\right)=0$ if $c \geq(g-1) / 2$. Hence we may assume $c \leq(g-3) / 2$. The following observation works for any $n$ with $1 \leq n \leq(g+1) / 2$ and all $P_{i}, 1 \leq i \leq n$, total ramification points of $f$ of type II.

Observation. We have $h^{0}\left(\omega_{X} \otimes\left(R^{\otimes(g-3) / 2}\right)^{\vee}\right)=1$ and $P_{1}+\cdots+P_{(g+1) / 2}$ is the only divisor in $\left|\omega_{X} \otimes\left(R^{\otimes(g-3) / 2}\right)^{\vee}\right|$, where $P_{1}, \ldots, P_{(g+1) / 2}$ denote all total ramification points of type $I I$ of $f$.

Proof of Observation. Duality gives $h^{0}\left(\omega_{X} \otimes\left(R^{\otimes(g-3) / 2}\right)^{\vee}\right)=1$. Let $D$ be the only divisor in $\left|\omega_{X} \otimes\left(R^{\otimes(g-3) / 2}\right)^{\vee}\right|$. We have $\operatorname{deg}(D)=(g+1) / 2$. Since each $P_{i}, 1 \leq i \leq(g+1) / 2$, is in the support of $D$, we get $D=P_{1}+\cdots+P_{(g+1) / 2}$.

(a) Assume $e_{1}=e_{2}=1$. First assume $c \leq(g-5) / 2$. Since $3 c+3 \in H\left(P_{2}\right)$ and $3 c+4 \in G\left(P_{2}\right), P_{2}$ is the base locus of $\left|\mathcal{O}_{X}\left(\left(a_{1}+2\right) P_{1}+a_{2} P_{2}\right)\right|$. Hence $a \notin H\left(P_{1}, P_{2}\right)$.

Now assume $c=(g-3) / 2$. The Observation gives $a \in H\left(P_{1}, P_{2}\right)$ and $h^{1}\left(\mathcal{O}_{X}\left(a_{1} P_{1}+a_{2} P_{2}\right)\right)=1$. 
(b) Assume $e_{1}=2$ and $e_{2}=1$. First assume $c \leq(g-5) / 2$. Since $3 c+3 \in$ $H\left(P_{2}\right)$ and $3 c+4 \in G\left(P_{2}\right), P_{2}$ is the base locus of $\left|\mathcal{O}_{X}\left(\left(a_{1}+1\right) P_{1}+a_{2} P_{2}\right)\right|$. Hence $a \notin H\left(P_{1}, P_{2}\right)$.

Now assume $c=(g-3) / 2$. We saw that $\left(a_{1}-1, a_{2}\right) \in H\left(P_{1}, P_{2}\right)$ and that $h^{1}\left(\mathcal{O}_{X}\left(\left(a_{1}-1\right) P_{1}+a_{2} P_{2}\right)\right)=1$. Hence if $a \in H\left(P_{1}, P_{2}\right)$, then $h^{1}\left(\mathcal{O}_{X}\left(a_{1} P_{1}+\right.\right.$ $\left.\left.a_{2} P_{2}\right)\right)=1$. Since $P_{2}$ has type II, we have $\left(a_{1}+1, a_{2}\right) \in H\left(P_{1}, P_{2}\right)$ and $h^{1}\left(\mathcal{O}_{X}\left(\left(a_{1}+1\right) P_{1}+a_{2} P_{2}\right)\right)=0$. Since $P_{1}$ is not in the base locus of $\mathcal{O}_{X}\left(\left(a_{1}+\right.\right.$ 1) $\left.P_{1}+a_{2} P_{2}\right)$, we get $h^{1}\left(\mathcal{O}_{X}\left(a_{1} P_{1} a_{2} P_{2}\right)\right)=0$ and hence $a \notin H\left(P_{1}, P_{2}\right)$.

(c) Assume $e_{1}=e_{2}=2$. First assume $c \leq(g-5) / 2$. Since $3 c+3 \in H\left(P_{2}\right)$ and $3 c+5 \in G\left(P_{2}\right), P_{2}$ is the base locus of $\left|\mathcal{O}_{X}\left(\left(a_{1}+1\right) P_{1}+a_{2} P_{2}\right)\right|$. Hence $a \notin H\left(P_{1}, P_{2}\right)$.

Now assume $c=(g-3) / 2$. We proved in Step (b) that $\left(a_{1}, a_{2}-1\right) \notin$ $H\left(P_{1}, P_{2}\right)$ and that $h^{1}\left(\mathcal{O}_{X}\left(\left(a_{1}, a_{2}-1\right)\right)=0\right.$. Therefore we have

$$
h^{1}\left(\mathcal{O}_{X}\left(a_{1} P_{1}, a_{2} P_{2}\right)\right)=0
$$

and

$$
h^{0}\left(\mathcal{O}_{X}\left(a_{1} P_{1}, a_{2} P_{2}\right)\right)=h^{0}\left(\mathcal{O}_{X}\left(\left(a_{1}-1\right) P_{1}+a_{2} P_{2}\right)\right)+1 .
$$

Therefore $P_{1}$ is not in the base locus of $\mathcal{O}_{X}\left(a_{1} P_{1}+a_{2} P_{2}\right)$. Taking $\left(P_{2}, P_{1}\right)$ instead of $\left(P_{1}, P_{2}\right)$ we get that $P_{2}$ is not in the base locus of $\mathcal{O}_{X}\left(a_{1} P_{1}+a_{2} P_{2}\right)$. Hence $a \in H\left(P_{1}, P_{2}\right)$.

Example 3. Let $X$ be a bielliptic curve of genus $g \geq 6$ and $f: X \rightarrow C$ its degree two covering of an elliptic curve $C . X$ and hence $f$ are uniquely determined by $C$, a line bundle $M \in \operatorname{Pic}^{g-1}(C)$ and and a reduced divisor $B \in\left|M^{\oplus 2}\right|$, say $B=O_{1}+\cdots+O_{2 g-2}$ (the branch locus). Let $P_{i}$ be the only point of $X$ with $f\left(P_{i}\right)=O_{i}$. We always take $n \leq 2 g-2$ and as $P_{1}, \ldots, P_{n}$ some of the points in the support of $B$, but not all $(M, B)$ gives semigroups with the same weight, we only choose the one with maximal weight. The semigroup $H\left(P_{i}\right)$ is known. Since $f^{-1}\left(O_{i}\right)=2 P_{i}$ (as schemes, all even integers $\geq 4$ are non-gaps of $P_{i}$. Obviously $1,2,3$ are gaps of $P_{i}$. Take an odd integer $k=2 m+1$ with $m \geq 2$. We have $f_{*}\left(\mathcal{O}_{X}\left(2 m P_{i}\right)\right)=f_{*}\left(f^{*}\left(\mathcal{O}_{C}\left(m O_{i}\right)\right) \cong \mathcal{O}_{C}\left(m O_{i}\right) \oplus \mathcal{O}_{C}\left(m O_{i}\right) \otimes M^{\vee}\right.$. Thus if $m>g-1$, then $h^{1}\left(\mathcal{O}_{X}\left((k-1) P_{i}\right)\right)=0$ and hence $k \in H\left(P_{i}\right)$ and $h^{1}\left(\mathcal{O}_{X}\left(k P_{i}\right)\right)=0$. Now assume $m<g-1$. Since $k-1, k+1 \in H\left(P_{i}\right)$ and $h^{0}\left(\mathcal{O}_{X}\left((k+1) P_{i}\right)=1+h^{0}\left(\mathcal{O}_{X}\left((k-1) P_{i}\right)\right)\right.$, we have $k \notin H\left(P_{i}\right)$. Now assume $c=g-1$. As in the last case we see that $k \in H\left(P_{i}\right)$ if and only if $h^{0}\left(\mathcal{O}_{X}\left((k+1) P_{i}\right)\right)=1+h^{0}\left(\mathcal{O}_{X}\left((k-1) P_{i}\right)\right)$, i.e. if and only if $(g-1) P_{i} \in|M|$. There are $2^{g-1}$ points $Q \in C$ such that $(g-1) Q \in|M|$. We fix $C$ and $M$. Since the linear system $\left|M^{\otimes 2}\right|$ induces an embedding $j: C \rightarrow \mathbb{P}^{2 g-3}$ and any $2 g-3$ 
points of $j(C)$ are linearly independent, for each integer $k \in\{0, \ldots, 2 g-4\}$ we may prescribe that $(g-1) P_{i} \in|M|$ if and only if $i \leq k$. We may also find $(X, f)$ associated to $(C, M)$ with $(g-1) P_{i} \in|M|$ for all $i$. From now on we take $n=2, k \geq 2$, and determine $H\left(P_{1}, P_{2}\right)$. Fix $\left(a_{1}, a_{2}\right) \in \mathbb{N}^{2}$ with $a_{1}>0$ and $a_{2}>0$. It is sufficient to do the case $4 \leq a_{1}+a_{2} \leq 2 g$. Write $a_{i}=2 b_{i}+e_{i}$ with $0 \leq e_{2} \leq e_{1} \leq 1$

(a) First assume $e_{1}=e_{2}=0$ and so $\left(a_{1}, a_{2}\right) \in H\left(P_{1}, P_{2}\right)$ and $h^{1}\left(\mathcal{O}_{X}\left(a_{1}+\right.\right.$ $\left.\left.a_{2} P_{2}\right)\right)=h^{1}\left(\mathcal{O}_{C}\left(b_{1} O_{1}+b_{2} O_{2}\right) \otimes M^{\vee}\right)$. If $b_{1}+b_{2} \neq g-1$, then the value of $h^{1}\left(\mathcal{O}_{X}\left(a_{1} P_{1}+a_{2} P_{2}\right)\right)$ only depends on $g$ and the integer $b_{1}+b_{2}$. If $b_{1}+b_{2}=g-1$ we also need to see if $b_{1} O_{1}+b_{2} O_{2} \in|M|$ or not (in the former case $h^{1}=1$, in the latter case $\left.h^{1}=0\right)$. By assumption $(g-1) P_{1} \in|M|,(g-1) P_{2} \in|M|$ and $P_{1} \neq P_{2}$. If $g-1$ is a prime, then no case with $b_{1}>0$ and $b_{2}>0$ may occur. If $g-1$ is not a prime, let $a$ be a proper factor of $g-1$ and assume that we take $P_{1}, P_{2}$ with $P_{1}-P_{2}$ of exact order $a$. Exactly $1+(g-1) / a$ of the divisors $b_{1} O_{1}+b_{2} O_{2}, b_{1} \geq 0, b_{2} \geq 0, b_{1}+b_{2}=g-1$, are elements of $|M|$. The only restriction to find $O_{1}, O_{2}$ for a fixed $C, M$ and $a$ is that $a$ divides $g-1$ and that $a \geq 2$. For larger $n$ we cannot find all $O_{1}, \ldots, O_{n}$ such that all pairs $\left(O_{i}, O_{j}\right)$, $i<j$, works for a proper divisor of $g-1$.

(b) Now assume $e_{1}=1$ and $e_{2}=0$. We have $\left(2 b_{1}, 2 b_{2}\right) \in H\left(P_{1}, P_{2}\right)$ and $\left(2 b_{1}+2, b_{2}\right) \in H\left(P_{1}, P_{2}\right)$. Hence $\left(a_{1}, a_{2}\right) \in H\left(P_{1}, P_{2}\right)$ if and only if $h^{0}\left(\mathcal{O}_{X}\left(\left(2 b_{1}+\right.\right.\right.$ 2) $\left.\left.P_{1}+2 b_{2} P_{2}\right)\right)=h^{0}\left(\mathcal{O}_{X}\left(2 b_{1} P_{1}+2 b_{2} P_{2}\right)\right)+2$, i.e. if and only if $h^{0}\left(\mathcal{O}_{C}\left(\left(b_{1}+1\right) P_{1}+\right.\right.$ $\left.\left.b_{2} P_{2}\right) \otimes M^{*}\right)>0$. Obviously this is the case if and only if either $b_{1}+b_{2} \geq g-1$ or $b_{1}+b_{2}=g-2$ and $\left(b_{1}+1\right) O_{1}+b_{2} O_{2} \in|M|$. We analyzed this case in part (a), (with the same $b_{2}$, but with $g-1-b_{2}$ as $\left.b_{1}\right)$. Note that if $\left(2 b_{1}+1, b_{2}\right) \in H\left(P_{1}, P_{2}\right)$, then our analysis gives that $\left(2 b_{1}, b_{2}+1\right) \notin H\left(P_{1}, P_{2}\right)$, because $O_{1} \neq O_{2}$. xms

(c) Now assume $e_{1}=e_{2}=1$. If $b_{1}+b_{2} \geq g-1$, then $a_{1}+a_{2} \geq 2 g$ and hence $\left(a_{1}, a_{2}\right) \in H\left(P_{1}, P_{2}\right)$ and $h^{1}\left(\mathcal{O}_{X}\left(a_{1} P_{1}+a_{2} P_{2}\right)\right)=0$.

Now assume $b_{1}+b_{2}=g-2$ and hence $a_{1}+a_{2}=2 g-2$. We have $h^{0}\left(\mathcal{O}_{X}\left(2 b_{1} P_{1}+2 b_{2} P_{2}\right)\right)=g-2$ and $h^{1}\left(\mathcal{O}_{X}\left(2 b_{1} P_{1}+2 b_{2} P_{2}\right)\right)=1$. Since $\left(a_{1}, a_{2}-1\right) \notin H\left(P_{1}, P_{2}\right)$ by part $(\mathrm{b})$, we have $h^{1}\left(\mathcal{O}_{X}\left(a_{1} P_{1}+\left(a_{2}-1\right) P_{2}\right)\right)=0$. Hence $\left(a_{1}, a_{2}\right) \in H\left(P_{1}, P_{2}\right)$ and $h^{1}\left(\mathcal{O}_{X}\left(a_{1}+a_{2} P_{2}\right)\right)=0$.

Assume $b_{1}+b_{2} \leq g-4$. The complete linear system $\mid \mathcal{O}_{X}\left(\left(a_{1}+1\right) P_{1}+\left(a_{2}+\right.\right.$ 1) $\left.P_{2}\right) \mid$ is induced by $f$, because $h^{0}\left(\mathcal{O}_{C}\left(\left(b_{1}+1\right) P_{1}+\left(b_{2}+1\right) P_{2}\right) \otimes M^{*}\right)=0$. Hence it has no divisor $E+P_{1}+P_{2}$ with $P_{1}$ not appearing in $E$. Hence $\left(a_{1}, a_{2}\right) \notin H\left(P_{1}, P_{2}\right)$.

Now assume $b_{1}+b_{2}=g-3$. We have $h^{0}\left(\mathcal{O}_{X}\left(2 b_{1} P_{1}+2 b_{2} P_{2}\right)\right)=g-3$. If $\left(b_{1}+1\right) O_{1}+\left(b_{2}+1\right) O_{2} \notin|M|$, then the linear system $\mid \mathcal{O}_{X}\left(\left(a_{1}+1\right) P_{1}+\right.$ $\left.\left(a_{2}+1\right) P_{2}\right) \mid$ is induced by $f$ and hence $\left(a_{1}, a_{2}\right) \notin H\left(P_{1}, P_{2}\right)$. Now assume 
$\left(b_{1}+1\right) P_{1}+\left(b_{2} P_{2}\right) \in|M|$. We are in the case analyzed in step (a), except now we need to check if $\left(a_{1}, a_{2}\right) \in H\left(P_{1}, P_{2}\right)$ or not. If $\left(b_{1}+1\right) P_{1}+\left(b_{2} P_{2}\right) \in|M|$, then $\mathcal{O}_{X}\left(\left(a_{1}+1\right) P_{1}+\left(a_{2}+1\right) P_{2}\right) \cong \omega_{X}$. Since $X$ is not hyperelliptic, $\omega_{X}$ is very ample and hence $h^{0}\left(\mathcal{O}_{X}\left(a_{1} P_{1}+a_{2} P_{2}\right)\right)=g-2$. We also get $h^{1}\left(\mathcal{O}_{X}\left(a_{1} P_{1}+a_{2} P_{2}\right)\right)=1$. Since $X$ is not trigonal, Serre duality gives that $\mathcal{O}_{X}\left(a_{1} P_{1}+a_{2} P_{2}\right)$ is spanned and hence $\left(a_{1}, a_{2}\right) \in H\left(P_{1}, P_{2}\right)$.

(d) In summary. Assume that either $g-1$ is prime and or that we took the semigroup $H\left(P_{1}, P_{2}\right)$ (not with maximal girst) with $O_{1}-O_{2}$ with exact order $g-1$. Then $H\left(P_{1}, P_{2}\right)$ and $v\left(P_{1}, P_{2}\right)$ is uniquely determined by $g$. If $g-1$ is composite, we may find 2-ples with higher gist. In each subcase to maximize the gist we need to take as $a$ the smallest prime divisor of $g-1$.

Example 4. Take $n=3, g \geq 2, X$ hyperelliptic and take $P_{1}, P_{2}, P_{3} \in X$ such that $P_{i} \neq P_{j}$ for all $i$ and $P_{1}+P_{2} \in g^{1_{2}}$. We have Fix $a=\left(a_{1}, a_{2}, a_{3}\right) \in \mathbb{N}^{3}$. We have $h^{0}\left(\mathcal{O}_{X}\left(a_{1} P_{1}+a_{2} P_{2}+a_{3} P_{3}\right)\right)=\max \left\{\min \left\{a_{1}, a_{2}\right\}+1, a_{1}+a_{2}+a_{3}+\right.$ $1-g\}$. If $a_{1}=a_{2}$ and $a_{3}=0$, then $h^{1}\left(\mathcal{O}_{X}\left(a_{1} P_{1}+a_{2} P_{2}+a_{3} P_{3}\right)\right)=\max \{0, g-$ $\min \left\{a_{1}, a_{2}\right\}$. Hence $a \in H\left(P_{1}, P_{2}, P_{3}\right)$ if and only if either $a_{1}=a_{2}$ and $a_{3}=0$ or $a_{1}+a_{2}+a_{3}-\min \left\{a_{1}, a_{2}\right\} \geq g+1-\min \left\{a_{1}, a_{2}\right\}$. Thus $a \in H\left(P_{1}, P_{2}, P_{3}\right)$ and $h^{1}\left(\mathcal{O}_{X}\left(a_{1} P_{1}+a_{2} P_{2}+a_{3} P_{3}\right)\right)>0$ if and only if $a_{1}=a_{2} \leq g-1$ and $a_{3}=0$. Therefore $v\left(P_{1}, P_{2}, P_{3}\right)=\sum_{i=1}^{g-1}(g-i)=g(g-1) / 2$.

\section{Acknowledgements}

The author was partially supported by MIUR and GNSAGA of INdAM (Italy).

\section{References}

[1] E. Ballico, S.J. Kim, Weierstrass multiple loci of $n$-pointed algebraic curves, J. Algebra, 199, No. 2 (1998), 455-471.

[2] C. Carvalho, T. Kato, On Weierstrass semigroups and sets: a review with new results, Geom. Dedicata, 139 (2009), 195-210.

[3] C. Carvalho, F. Torres, On Goppa codes and Weierstrass gaps at several points, Des. Codes Cryptogr., 35, No. 2 (2005), 211-225.

[4] M. Coppens,The Weierstrass gap sequences of the total ramification points of trigonal coverings of $\mathbb{P}^{1}$, Nederl. Akad. Wetensch. Indag. Math., 47, No. 3 (1985), 245-276. 
[5] T. Kato, K. Magaard, H. Völklein, Indag. Math. (N.S.), 22, No-s: 1-2 (2011), 116-130.

[6] S.J. Kim, On the index of the Weierstrass semigroup of a pair of points on a curve, Arch. Math., 62 (1994), 73-82.

[7] G. Martens, F.-O. Schreyer, Line bundles and syzygies of trigonal curves, Abh. Math. Sem. Univ. Hamburg, 56 (1986), 169-189.

[8] J. Park, A Note on Weierstrass points of bielliptic curves, Manuscripta Math., 95 (1998), 33-45. 